Romero-Díaz et al.

Coexistencia y segregación trófica de aves Ecosist. Recur. Agropec.

$5(15): 477-489,2018$

\title{
Coexistencia y segregación trófica en aves insectívoras de un bosque templado con tres elevaciones
}

\section{Coexistence and trophic segregation in insectivorous birds of a temperate forest with three elevations}

\author{
Claudio Romero-Díaz ${ }^{1}$, Saúl Ugalde-Lezama ${ }^{2 *}$, Luis Antonio Tarango-Arámbula ${ }^{3}$, Víctor Manuel Ruíz-Vera ${ }^{3}$, \\ Uriel Marcos-Rivera ${ }^{1}$, Yessenia Cruz-Miranda ${ }^{2}$ \\ ${ }^{1}$ Instituto Tecnológico Superior de Zacapoaxtla. Km 8, Carretera Acuaco-Zacapoaxtla, CP. 73680. Totoltepec, Zacapoaxtla, \\ Puebla, México. \\ ${ }^{2}$ Departamento de Suelo, Universidad Autónoma Chapingo. Km 38, Carretera México-Texcoco, CP. 56230. Chapingo, Estado \\ de México, México. \\ ${ }^{3}$ Colegio de posgraduados Campus San Luis Potosí, Agustín de Iturbide No 73, CP. 78600. Salinas de Hidalgo, Salinas, San \\ Luis Potosí, México. \\ ${ }^{*}$ Autor de correspondencia: biologo_ugalde@hotmail.com
}

Artículo científico recibido: 07 de agosto de 2017 aceptado: 22 de mayo de 2018

RESUMEN. Con el objetivo de inferir patrones de coexistencia y segregación trófica para aves de bosque templado en tres elevaciones de la Cuenca de San Bernardino, Estado de México. Se realizó el seguimiento trófico de aves insectívoras de octubre de 2016 a enero de 2017. Se emplearon corte-sacudida de ramas, búsqueda Intensiva, redes de niebla, análisis de excretas e identificación de insectos. Se utilizaron análisis de frecuencia de observación (Fo), regresión de Poisson (ARP), $\mathrm{X}^{2}$; Kruskal-Wallis y correspondencia simple (ACS). El valor general promedio para Fo señala que la técnica de cacería más utilizada es colectar ( $F o=91 \%)$ y para ARP colectar y remover $(\operatorname{Pr}>Z=-7.291$, 0.929). Los órdenes de insectos consumidos son Coleóptera e Hymenoptera ( $\operatorname{Pr}>Z=<13$, 6.959). La $X^{2}$ muestra que lo registrado es similar a lo esperado (Prob $>$ Chisq $=0.5721,0.2474,0.2956,0.508$ 9). Kruskal-Wallis evidencia que las técnicas de cacería-dieta son similares (Prob > Ji cuadrado $=0.5371,0.2622,0.1809,0.4665$ ). El ACS muestra coexistencia en la formación de cuatro grupos avifaunísticos (Inercia $=100,47.87,28.84,83.15 \%$; técnicas de cacería, ordenes, familias, tipos de insectos, respectivamente). La altitud no es un factor determinante de coexistencia trófica, ya que no afecta la disponibilidad de presas, por lo que no modifica las técnicas de cacería utilizadas por las aves, exhibiendo dietas similares, con simpátria trófica. Se recomiendan estrategias de mejoramiento de hábitat que promuevan la disponibilidad de presas para éste tipo de aves.

Palabras clave: Avifauna, muestreo-sistemático, San Bernardino

ABSTRACT. In order to infer patterns of coexistence and trophic segregation for temperate forest birds in three elevations of the San Bernardino Basin, State of Mexico, trophic tracking of insectivorous birds was carried out from October 2016 to January 2017. Cut-shaking of branches, intensive searches, mist nets, excreta analysis and insect identification were used. Frequency of observation analysis (Fo), Poisson regression (PRA), $X^{2}$, the Kruskal-Wallis test and simple correspondence analysis (SCA) were used. The overall average value for Fo indicates that the most used hunting technique is to collect $(\mathrm{Fo}=91 \%)$ and for $\mathrm{PRA}$ to collect and remove ( $\operatorname{Pr}>Z=-7.291,0.929)$. The orders of insects consumed are Coleoptera and Hymenoptera ( $\operatorname{Pr}>Z=<13,6.959)$. The $X^{2}$ shows that what was recorded is similar to what was expected (Prob > Chisq $=0.5721,0.2474,0.2956,0.508$ 9). Kruskal-Wallis shows that hunting-diet techniques are similar (Prob $>$ Chi square $=0.5371,0.2622,0.1809,0.4665$ ). The SCA shows coexistence in the formation of four avifaunal groups (Inertia $=100,47.87,28.84,83.15 \%$; hunting techniques, orders, families, types of insects, respectively). Elevation is not a determinant of trophic coexistence, since it does not affect the availability of prey, so it does not modify the hunting techniques used by birds, exhibiting similar diets, with trophic 
Romero-Díaz et al.

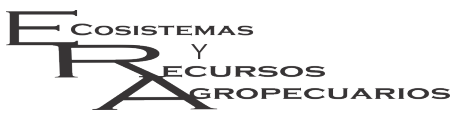

Coexistencia y segregación trófica de aves Ecosist. Recur. Agropec.

$5(15): 477-489,2018$

sympatry. Habitat improvement strategies that promote the availability of prey for this type of bird are recommended. Key words: Avifauna, systematic sampling, San Bernardino

\section{INTRODUCCIÓN}

A nivel mundial se tiene un registro de 9271 especies de aves (Navarro-Sigüenza et al. 2014). Pero a pesar de esta gran diversidad de especies las tazas de extinción han incrementado de forma drástica debido a la destrucción y alteración de su hábitat, que altera los procesos ecológicos positivos y negativos, como la segregación de nichos y la competencia por nuevos espacios (Birdlife International 2008). Durante la obtención del alimento, las aves emplean diferentes técnicas para la obtención del recurso, basado en gremios tróficos y competencia por alimento (Torrens et al. 2017). La teoría del forrajeo óptimo menciona que las aves utilizan técnicas de cacería que requieren menor gasto energético para obtener su alimento, con mayor aporte nutricional (Ginnobili y Roffe 2016, Cruz-Miranda et al. 2017). De igual forma el comportamiento de los organismos se define por la selección natural, misma que se integra por eventos y coacciones que determinan la supervivencia de las especies, y determinan el éxito reproductivo (Frere et al. 2005). La hipótesis del gasto de crianza indica que las aves residentes, en época reproductiva estan limitadas por la presencia de pocos artrópodos de talla grande, mismas que capturan para optimizar su adecuación reproductiva, mientras que en periodo no reproductivo se tiene abundancia de presas de menor tamaño que ayuda a los organismos adultos a sobrevivir (Johnson et al. 2005, Fandiño et al. 2016). A partir de diversas estrategias las aves demuestran una utilización del hábitat que les permite establecer interacciones positivas (Velasco et al. 2017, Seeholzer et al. 2017). Un aspecto importante que determina la coexistencia de aves insectívoras es la distribución de sus presas, lo cual puede determinar la ecología de las aves insectívoras (De la Ossa et al. 2017).

En México se registran 1107 especies de aves, de las cuales 102 son endémicas (Berlanga et al. 2015), que se distribuyen desde zonas tropicales hasta semiáridas, encontrándose algunas en alguna categoría de riesgo (SEMARNAT 2010). Debido a una amplia gama de factores de perturbación y disturbio (Navarro-Sigüenza et al. 2014), lo que ha provocado diversos procesos de segregación por espacio, alimento y dominio de nuevos nichos ecológicos (Pineda-Pérez et al. 2014). Por ello las aves insectívoras tienen fluctuaciones, debido a la estacionalidad de insectos, viéndose obligadas a recurrir a las siguientes acciones: cambiar su dieta, consumir insectos inactivos o abandonar el área y buscar un nuevo nicho alimenticio (Ortiz-Pulido et al. 2016).

Una zona con un rodal forestal relevante para aves insectívoras en bosque templado de pinoencino, se localiza en la Cuenca San Bernardino, en la parte central del Eje Neo volcánico transversal; sujeta a perturbación como la tala inmoderada, urbanización, cacería furtiva, tráfico ilegal y el cambio de uso de suelo (Moreno 2007). En general, las aves juegan un papel importante en el control biológico y equilibrio del ambiente, por lo que se consideran bioindicadoras de perturbación (Navarro-Sigüenza et al. 2014); forman parte de cadenas tróficas, y son dispersoras de semillas y polinizadoras (Aguilar y Tinoco 2017). A pesar de su importancia ecológica, en México existen pocos estudios sobre ecología trófica de aves (Ugalde-Lezama et al. 2011 y PinedaPérez et al. 2014), que reportan la determinación de dietas de aves insectívoras de selvas y bosques templados. Por lo anterior, el objetivo del presente trabajo fue determinar de forma comparativa las dietas y coexistencia trófica en la estructura de aves insectivoras de un bosque de pino-encino en tres elevaciones de la Cuenca de San Bernardino, Texcoco, Estado de México.

\section{MATERIALES Y MÉTODOS}

\section{Área de estudio}

La Subcuenca del río San Bernardino se ubica en el municipio de Texcoco de Mora, Estado de México. En la parte este del monte Tláloc, a una altitud de entre 2600 y $3000 \mathrm{msnm}$, en las 
Romero-Díaz et al.

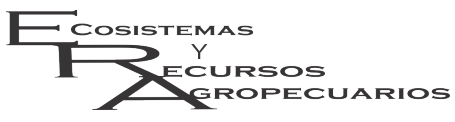

Coexistencia y segregación trófica de aves Ecosist. Recur. Agropec.

5(15):477-489,2018

coordenadas $98^{\circ} 4744.19$ y $98^{\circ} 4520.85$ de LN y $19^{\circ} 280.91$ y $19^{\circ} 2629.68$ LO. Se evaluaron las elevaciones altitudinales (EA) de : $2600 \mathrm{msnm}$ (EA2600), $2800 \mathrm{msnm}$ (EA2800) y $3000 \mathrm{msnm}$ (EA3000) considerando una superficie de 21 ha para cada elevación. En cada EA se usó muestreo sistemático (MS) con distancias predeterminadas de $200 \mathrm{~m}$ en arreglos a manera de cuadriculas, con tamaño de muestra de ocho unidades de elección (UEL) con una superficie de $50 \mathrm{~m}^{2}$ cada uno y 200 $\mathrm{m}$ de distancia entre cada punto (Gallina y LópezGonzález 2011). Cada 16 d se realizó el seguimiento de las aves de octubre de 2016 a enero de 2017, aplicando un total de 5 muestreos. El monitoreo se realizó en función del muestreo de recuento en puntos con radio fijo y búsqueda intensiva. Este esquema consistió en que el observador al llegar a cada punto de muestreo, se mantenía quieto por un minuto y luego recorría un radio de $25 \mathrm{~m}$ en búsqueda de ejemplares, utilizando binoculares de 8 x $42 \mathrm{~m}$ de resolución marca Bushnell. Únicamente se registraron individuos que se encontraban alimentando o aplicando alguna técnica de cacería, de las 07:00 a 14:00 $\mathrm{h}$, periodo en que las aves presentan mayor actividad forrajera.

El monitoreo de insectos se realizó con el método de corte y sacudida de ramas, que consiste en ubicar a las aves alimentándose. Para luego colocar una bolsa de plástico en la rama en la que se encontraba el ave y sacudir con el fin de obtener los insectos de los que potencialmente se pudiera estar alimentando. La identificación de los insectos se realizó con claves taxonómicas propuestas por Borror et al (1989), Equihua (1989) y Stehr (1991). Los insectos se fotografiaron con una cámara digital marca Pax Cam 3, con óptica Kern Macro-Switar, modelo profesional para equipos de microscopia electrónica de barrido marca JEOL 35$C$ y se medieron con el analizador de imágenes Image Tool para Windows versión 3.0. Para la obtención de excretas se llevó a cabo la contención física de aves mediante el método de captura con redes de niebla mediante esfuerzo constante (Villaseñor y Santana 2002). Las redes utilizadas tuvieron un tamaño de $12 \mathrm{~m}$ de largo por $2.5 \mathrm{~m}$ de ancho y luz de malla de $36 \mathrm{~mm}$ de diámetro, se colacaron de las 07:00 a 16:00 h. Las aves capturadas se colocaron en cajas de cartón por una hora con la finalidad de obtener sus heces, las cuales se colocaron en viales con alcohol al $70 \%$ y se trasladaron al laboratorio de entomología de la Universidad Autónoma Chapingo. Las muestras se analizaron con la técnica propuesta por Whitaker (1988) con un microscopio estereoscopio con resolución de 10 a 80 x. Los fragmentos encontrados se identificaron con las claves taxonómicas antes señaladas; mientras que la identificación de las aves se realizó con las guías de identificación estándar (Peterson y Chalif 1989, National Geographic 2002).

Para determinar la posible asociación entre las aves e insectos derivados de la técnica de corte y sacudida de ramas, las técnicas de cacería y los insectos consumidos; se aplicaron análisis de regresión de poisson (ARP), con el procedimiento de selección de variables polinómica por pasos hacia atrás (Stepwise), el ajuste de los modelos se realizó con el criterio del mínimo Akaike en R.13.0 (Akaike 1969). Para establecer posibles diferencias estadísticas en los datos registrados en las diferentes elevaciones, e inferir si los datos registrados son los potencialmente existentes, se aplicaron análisis de $x^{2}$ y Krustal Wallis, en virtud de no cumplirse los supuestos de la estadística paramétrica, los análisis se realizaron con el programa JMP IN versión 8.0. Para visualizar de forma gráfica la posible relación entre las aves y las variables evaluadas, se realizaron análisis de correspondencia simple (ACS), con el programa Statistica ${ }^{T M}$ versión 13.0.

\section{RESULTADOS}

Los resultados de la regresión de Poisson para los datos de corte y sacudida de ramas sugieren que siete familias de insectos presentan efecto sobre las abundancias de aves en las elevaciones consideradas, presentando un AIC de 65.691, 57.811, 106.08 y 87.63 (Tabla 1). Mientras que las técnicas de cacería sugieren que una técnica presenta efecto sobre la abundancia de aves en las elevaciones, con AIC de 28.735, 25.21, 32.823 y 67.65 (Tabla 2). 
Romero-Díaz et al.

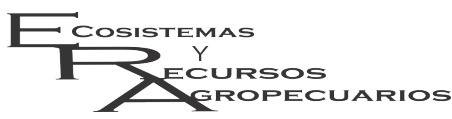

Coexistencia y segregación trófica de aves

Ecosist. Recur. Agropec.

$5(15): 477-489,2018$

Tabla 1. Resultados de regresión Poisson para las abundancias de las familias de insectos registradas en cada elevación.

\begin{tabular}{|c|c|c|c|c|c|}
\hline \multicolumn{6}{|c|}{ Elevación altitudinal 2600} \\
\hline Coeficientes: & Estimados & Error Estándar & Valor de Z & $\operatorname{Pr}(>|Z|)$ & Significancia \\
\hline (Intercepto) & -1.02962 & 0.44721 & -2.302 & 0.021316 & $*$ \\
\hline Anthocoridae & 0.10562 & 0.01423 & 7.421 & -11.831 & $* * *$ \\
\hline Bostrichidae & 0.28713 & 0.13944 & 2.059 & 0.039484 & $*$ \\
\hline Cicadellidae & 0.37701 & 0.09035 & 4.173 & -1.891 & $* * *$ \\
\hline Coccinellidae & 1.31953 & 0.31622 & 4.173 & -1.891 & $* * *$ \\
\hline Geometridae & 0.48318 & 0.13416 & 3.601 & 0.000316 & $* * *$ \\
\hline Membracidae & 0.57426 & 0.27889 & 2.059 & 0.039484 & * \\
\hline Miridae & 0.60398 & 0.1677 & 3.601 & -0.831 & $* * *$ \\
\hline No identificado & 0.20168 & 0.03486 & 5.785 & -1.751 & $* * *$ \\
\hline \multicolumn{6}{|c|}{ Elevación altitudinal 2800} \\
\hline (Intercepto) & -2.3979 & 1 & -2.398 & 0.01649 & * \\
\hline Anthocoridae & 2.3979 & 1.41421 & 1.696 & 7.009 & . \\
\hline Cicadellidae & 0.40073 & 0.10954 & 3.658 & 0.000254 & $* * *$ \\
\hline Coccinellidae & 1.19895 & 0.70711 & 1.696 & 7.009 & . \\
\hline Eulophidae & 2.3979 & 1.41421 & 1.696 & 7.009 & . \\
\hline Gelechiidae & 0.7993 & 0.4714 & 1.696 & 0.089968 & . \\
\hline Largidae & 2.3979 & 1.41421 & 1.696 & 0.089968 & . \\
\hline Lumbricidae & 1.19895 & 0.70711 & 1.696 & 7.009 & \\
\hline Miridae & 1.33578 & 0.36515 & 3.658 & -1.451 & $* * *$ \\
\hline No identificado & 0.35347 & 0.08108 & 4.359 & -3.691 & $* * *$ \\
\hline Phoridae & 2.3979 & 1.41421 & 1.696 & 7.009 & . \\
\hline Tephritidae & 2.3979 & 1.41421 & 1.696 & 7.009 & . \\
\hline \multicolumn{6}{|c|}{ Elevación altitudinal 3000} \\
\hline (Intercept) & 0.699 & 1.369 & 0 & 1 & \\
\hline Cicadellidae & 7.059 & 2.539 & 3.184 & -1.541 & $* *$ \\
\hline Geometridae & 2.309 & 1.049 & 10.832 & $<2 \mathrm{E}-16$ & $* * *$ \\
\hline Lauxaniidae & 2.30 & 2.949 & 5.838 & -3.721 & $* * *$ \\
\hline No identificado & 0.819 & 0.339 & 7.746 & -5.531 & $* * *$ \\
\hline \multicolumn{6}{|c|}{ Todas las elevaciones } \\
\hline (Intercept) & 0.251314 & 0.235702 & 1.066 & 0.28632 & \\
\hline Anthocoridae & 0.065686 & 0.008022 & 8.188 & -13.331 & $* * *$ \\
\hline Cicadellidae & 0.129302 & 0.0184 & 7.027 & -9.881 & $* * *$ \\
\hline Coccinellidae & 0.385111 & 0.117851 & 3.268 & -1.911 & $* *$ \\
\hline Geometridae & 0.212178 & 0.019756 & 10.74 & $<2 \mathrm{E}-16$ & $* * *$ \\
\hline Lauxaniidae & 2.051271 & 0.394405 & 5.201 & -5.011 & $* * *$ \\
\hline Miridae & 0.277987 & 0.058321 & 4.766 & -4.121 & $* * *$ \\
\hline No identificado & 0.073754 & 0.006659 & 11.075 & $<2 \mathrm{E}-16$ & $* * *$ \\
\hline Tephritidae & 0.477549 & 0.07127 & 6.701 & -8.911 & $* * *$ \\
\hline
\end{tabular}

Para el consumo de insectos se observa que un orden de insectos tiene efecto sobre las abundancias de las aves con AIC de 25.159, 19.909, 18.755 y 33.503 (Tabla 3). Los datos de corte y sacudida de ramas derivados del ARP indican que no existen diferencias significativas entre elevaciones (Tabla 4). Para las técnicas de cacería colectar y remover, no se observaron diferencias significativas entre ellas (Tabla 5). Los insectos consumidos (excretas) Coleóptera e Hymenoptera, no presentan diferencias significativas entre elevaciones, por loque son estadísticamente similares. Los resultados de $\mathrm{X}^{2}$ para los datos de corte y sacudida de ramas derivados del ARP, sugieren que las familias de insectos registradas son las que potencialmente podrían presentarse en las elevaciones estudiadas, a excepción de Miridae (Tabla 6). Las técnicas de cacería colectar y remover son las que pueden ser exhibidas por las aves (Tabla 7), mientras que los insectos de los órdenes Coleóptera e Hymenoptera son los más consumidos por la avifauna.

Los resultados del ACS para corte y sacudida de ramas derivados del ARP muestran una asociación en la inercia total $(28.84 \%)$, recono- 
Romero-Díaz et al.

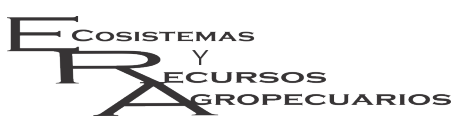

Coexistencia y segregación trófica de aves

Ecosist. Recur. Agropec.

$5(15): 477-489,2018$

Tabla 2. Resultados de regresión Poisson para las abundancias de aves y las técnicas de cacería exhibidas en cada elevación.

\begin{tabular}{|c|c|c|c|c|c|}
\hline \multicolumn{6}{|c|}{ Elevación altitudinal 2600} \\
\hline Coeficientes: & Estimados & Error Estándar & Valor de Z & $\operatorname{Pr}(>|Z|)$ & Significancia \\
\hline (Intercepto) & 0.09954 & 0.34719 & 0.287 & 0.774 & \\
\hline Colectar & 0.22777 & 0.05032 & 4.527 & 0.009 & $* * *$ \\
\hline \multicolumn{6}{|c|}{ Elevación altitudinal 2800} \\
\hline (Intercepto) & -0.572 & 0.9963 & -0.574 & 0.566 & \\
\hline Remover & 0.4908 & 0.2876 & 1.706 & 6.809 & . \\
\hline \multicolumn{6}{|c|}{ Elevación altitudinal 3000} \\
\hline (Intercepto) & 0.7062 & 0.306 & 2.308 & 0.021 & \\
\hline Colectar & 0.1546 & 0.0372 & 4.156 & -1.761 & $* * *$ \\
\hline \multicolumn{6}{|c|}{ Elevaciones 2600-3000 } \\
\hline (Intercepto) & 0.4153 & 0.23413 & 1.774 & 0.0761 & \\
\hline Colectar & 0.18576 & 0.02941 & 6.315 & -7.291 & $* * *$ \\
\hline Remover & 0.23548 & 0.10801 & 2.18 & 0.929 & $*$ \\
\hline
\end{tabular}

Tabla 3. Resultados de Regresión Poisson para la abundancia de aves y los órdenes de insectos registrados en las excretas de cada elevación.

\begin{tabular}{|c|c|c|c|c|c|}
\hline \multicolumn{6}{|c|}{ Elevación altitudinal 2600} \\
\hline Coeficientes: & Estimados & Error Estándar & Valor de Z & $\overline{\operatorname{Pr}(>|\mathrm{Z}|)}$ & Significancia \\
\hline (Intercepto) & 0.470004 & 0.353553 & 1.329 & 0.184 & \\
\hline Coleóptera & 0.041604 & 0.006553 & 6.348 & -7.821 & $* * *$ \\
\hline \multicolumn{6}{|c|}{ Elevación altitudinal 2800} \\
\hline (Intercepto) & -0.223144 & 0.499999 & -0.446 & 0.655 & \\
\hline Coleóptera & 0.048649 & 0.008637 & 5.633 & -6.211 & $* * *$ \\
\hline \multicolumn{6}{|c|}{ Elevación altitudinal 3000} \\
\hline (Intercepto) & -1.386294 & 0.999932 & -1.386 & 0.1656 & \\
\hline Coleóptera & 0.038083 & 0.007865 & 4.842 & -4.711 & $* * *$ \\
\hline Himenóptera & 0.231049 & 0.136077 & 1.698 & 6.959 & \\
\hline \multicolumn{6}{|c|}{ Elevaciones 2600-3000 } \\
\hline (Intercepto) & 1.098612 & 0.258199 & 4.255 & -2.901 & $* * *$ \\
\hline Coleóptera & 0.012521 & 0.001106 & 11.32 & $<2 \mathrm{e}-16$ & $* * *$ \\
\hline
\end{tabular}

ciendo la conformación de tres grupos de aves que hacen uso de las familias de insectos registrados (Figura 1). Las técnicas de colectar y remover tuvieron sociación, mostrando la conformación de tres conjuntos avifaunísticos que hacen uso de estas técnicas (Figura 2). El consumo de insectos señala asociación (47.87\%), lo que indica la conformación de cuatro bloques de aves que consumen estos insectos (Figura 3). Lo que sugiere que, durante su alimentación, las aves establecen estrategias tróficas que les permite tener dietas diferenciales a partir de la utilización de los insectos disponibles.

\section{DISCUSIÓN}

La tendencia de las técnicas de cacería empleadas por las aves en este estudio, coincide con lo reportado por Pineda-Pérez et al. (2014) en un estudio sobre ecología trófica de aves insectívoras, quienes mencionan que varias especies avifaunísticas presentan pico grueso, lo que les permite capturar coleópteros. Esto ha sido confirmado por Colorado (2004) quien comprobó que la morfología del pico y de las patas explican los insectos que capturan las aves. Los patrones de cacería exhibidos concuerdan con lo reportado por Adamik y Korñan (2004) quienes mencionan que las aves se alimentan de presas disponibles en la corteza, empleando la técnica de colectar. Sobre lo mismo Cueto y López de Casanave (2002) reportan que las aves Passerinas utilizan las técnicas de colectar y revoloteo. Mientras que Caicedo-Argüelles y Cruz-Bernate (2014), señalan que la alimentación es la actividad en que las aves invierten más tiempo y las técni- 
Romero-Díaz et al.

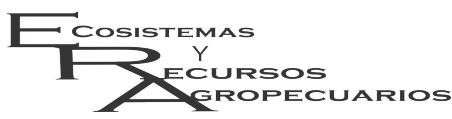

Coexistencia y segregación trófica de aves

Ecosist. Recur. Agropec.

$5(15): 477-489,2018$

Tabla 4. Resultados de Kruskal-Wallis para las familias de insectos, derivadas del $A R P$, en las elevaciones 2600, 2800, 3000 en la cuenca de San Bernardino, Texcoco, Estado de México.

\begin{tabular}{lccc}
\hline \multicolumn{4}{c}{ Familias de insectos datos de presencias y ausencias } \\
\hline Familia & Ji cuadrado & Grados de libertad & Prob $>$ Ji cuadrado \\
Anthocoridae & 3.5 & 1 & 0.0614 \\
Bostrichidae & 3.2308 & 1 & 0.0723 \\
Cicadellidae & 0 & 1 & 1 \\
Coccinellidae & 0.8077 & 1 & 0.3688 \\
Geometridae & 0 & 1 & 1 \\
Membracidae & 3.2308 & 1 & 0.0723 \\
Miridae & 6.2222 & 1 & $0.0126^{*}$ \\
No identificado & 0.5833 & 1 & 0.445 \\
Eulophidae & 0 & 1 & 1 \\
Gelechiidae & 0 & 1 & 1 \\
Largidae & 0 & 1 & 1 \\
Lumbricidae & 0 & 1 & 1 \\
Phoridae & 0 & 1 & 1 \\
Tephritidae & 0.4773 & 1 & 0.4897 \\
Lauxaniidae & 1.5 & 1 & 0.2207 \\
& Familias de insectos datos de frecuencias & \\
Anthocoridae & 7.4667 & 4 & 0.1132 \\
Bostrichidae & 3.2308 & 1 & 0.0723 \\
Cicadellidae & 3.9667 & 4 & 0.4105 \\
Coccinélida & 1.5077 & 2 & 0.4706 \\
Geometridae & 4.6667 & 4 & 0.3232 \\
Membracidae & 3.2308 & 1 & 0.0723 \\
Miridae & 6.9689 & 2 & $0.0307 *$ \\
No identificado & 4.9 & 5 & 0.4282 \\
Eulophidae & 0 & 1 & 1 \\
Gelechiidae & 0 & 1 & 1 \\
Largidae & 0 & 1 & 1 \\
Lumbricidae & 0 & 1 & 0.3046 \\
Phoridae & 0 & 1 & \\
Tephritidae & 3.6273 & 1 & 1 \\
Lauxaniidae & 1.5 & & \\
\hline & & 1 & \\
\hline
\end{tabular}

Tabla 5. Resultados de Kruskal-Wallis para colectar y remover, Coleóptera e Hymenoptera derivadas del $A R P$, en las elevaciones 2600, 2800, 3000 de la cuenca de San Bernardino Texcoco Estado de México.

\begin{tabular}{|c|c|c|c|}
\hline \multicolumn{4}{|c|}{ Técnicas de cacería datos de presencia ausencia } \\
\hline Técnica & Ji cuadrada & Grados de libertad & Prob > Ji cuadrado \\
\hline Colectar & 0.0635 & 1 & 0.8011 \\
\hline Remover & 1.2574 & 1 & 0.2622 \\
\hline \multicolumn{4}{|c|}{ Técnicas de cacería datos frecuencias } \\
\hline Colectar & 3.1252 & 4 & 0.5371 \\
\hline Remover & 1.2574 & 1 & 0.2622 \\
\hline \multicolumn{4}{|c|}{ Ordenes de insectos } \\
\hline Coleóptera & 8.8716 & 6 & 0.1809 \\
\hline Hymenoptera & 0.5303 & 1 & 0.4665 \\
\hline
\end{tabular}

cas de alimentación que utilizan son recoger y capturar. En contraste, Camphuysen y Webb (1999) y Ostrand (1999) mencionan que las aves integran grupos de alimentación, como resultado de la conglomeración de insectos, lo que ceba a predadores que interactúan entre sí; pero de acuerdo con Mills (1998) éste comportamiento se explica a través del reclutamiento visual en el que las aves que se alimentan atraen a otras, por lo que funcionan como indicadoras catalíticas.

Los órdenes de insectos consumidos concuerdan con lo reportado por Perovic et al. (2018) en un estudio de la dieta de aves insectívoras, como controladoras de plagas, que consumieron 
Romero-Díaz et al.

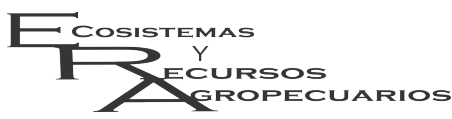

Coexistencia y segregación trófica de aves

Ecosist. Recur. Agropec.

$5(15): 477-489,2018$

Tabla 6. Análisis de $\mathrm{X}^{2}$ para las Familias de insectos probablemente colectadas por las aves durante su alimentación en las elevaciones bajo estudio.

\begin{tabular}{|c|c|c|c|c|}
\hline \multicolumn{5}{|c|}{ Familias de insectos datos de presencia ausencia } \\
\hline Familia & $\mathrm{N}$ & Grados de libertad & Ji cuadrado & Prob $>$ Ji Cuadrado \\
\hline Anthocoridae & 15 & 2 & 5 & 0.0821 \\
\hline Bostrichidae & 15 & 2 & 4.615 & 0.0995 \\
\hline Cicadellidae & 15 & 2 & 0.536 & 0.765 \\
\hline coccinellidae & 15 & 2 & 1.154 & 0.5616 \\
\hline Geometridae & 15 & 2 & 5 & 0.0821 \\
\hline Membracidae & 15 & 2 & 4.615 & 0.0995 \\
\hline Miridae & 15 & 2 & 6.667 & $0.357^{*}$ \\
\hline No identificado & 15 & 2 & 2.5 & 0.2865 \\
\hline Eulophidae & 15 & 2 & 2.143 & 0.3425 \\
\hline Gelechiidae & 15 & 2 & 2.143 & 0.3425 \\
\hline Largidae & 15 & 2 & 2.143 & 0.3425 \\
\hline Lumbricidae & 15 & 2 & 2.143 & 0.3425 \\
\hline Phoridae & 15 & 2 & 2.143 & 0.3425 \\
\hline Tephritidae & 15 & 2 & 0.682 & 0.7111 \\
\hline Lauxaniidae & 15 & 2 & 2.143 & 0.3425 \\
\hline \multicolumn{5}{|c|}{ Familias de insectos datos de frecuencias } \\
\hline Anthocoridae & 15 & 8 & 10 & 0.265 \\
\hline Bostrichidae & 15 & 2 & 4.615 & 0.0995 \\
\hline Cicadellidae & 15 & 8 & 7.25 & 0.5099 \\
\hline Coccinellidae & 15 & 4 & 4.154 & 0.3856 \\
\hline Geometridae & 15 & 8 & 10 & 0.265 \\
\hline Membracidae & 15 & 2 & 4.615 & 0.0995 \\
\hline Miridae & 15 & 4 & 9.867 & $0.0427^{*}$ \\
\hline No identificado & 15 & 10 & 12 & 0.2851 \\
\hline Eulophidae & 15 & 2 & 2.143 & 0.3425 \\
\hline Gelechiidae & 15 & 2 & 2.143 & 0.3425 \\
\hline Largidae & 15 & 2 & 2.143 & 0.3425 \\
\hline Lumbricidae & 15 & 2 & 2.143 & 0.3425 \\
\hline Phoridae & 15 & 2 & 2.143 & 0.3425 \\
\hline Tephritidae & 15 & 6 & 5.182 & 0.5207 \\
\hline Lauxaniidae & 15 & 2 & 2.143 & 0.3425 \\
\hline
\end{tabular}

Tabla 7. Resultados de los análisis de $X^{2}$ para las técnicas de cacería y ordenes de insectos consumidos por las aves durante su alimentación.

\begin{tabular}{ccccc}
\hline \multicolumn{5}{c}{ Datos de presencia ausencia } \\
\hline Técnica & $\mathrm{N}$ & Grados de libertad & Ji Cuadrada & Prob > Ji Cuadrada \\
Colectar & 22 & 2 & 2.245 & 0.3255 \\
Remover & 22 & 2 & 2.794 & 0.2474 \\
& \multicolumn{5}{c}{ Datos de frecuencia } \\
Colectar & 22 & 8 & 6.675 & 0.5721 \\
Remover & 22 & 2 & 2.794 & 0.2474 \\
& \multicolumn{5}{c}{ Ordenes de insectos consumidos } \\
Coleóptera & 34 & 12 & 14.081 & 0.2956 \\
Hymenoptera & 34 & 2 & 1.351 & 0.5089 \\
\hline
\end{tabular}

Coleópteros e Himenópteros. En la evaluación de la alimentación de aves insectívoras en un bosque de coníferas los órdenes de insectos más consumidos fueron Coleóptera e Hymenoptera (Haro 1998). Lo que concuerda con Rosas-Espinoza et al. (2008) quienes estudiaron la dieta de Melanerpes formicivorus, encontrando que además de bellotas de Quercus castanea, $Q$. viminea, $Q$. resinosa y Pinus magnoliifolia, también consumen insectos de los ordenes Coleóptera y Lepidóptera, además de savia de árboles. Por su lado, Recher y Majer (2006) evaluaron el efecto de la exclusión de aves mediante la depredación de Artrópodos de dosel en un bosque de eucalipto, encontrando que los Coleópteros y Lepidópteros larvales son los más consumidos debido a su alta disponibilidad, por lo que dichas aves 
Romero-Díaz et al.

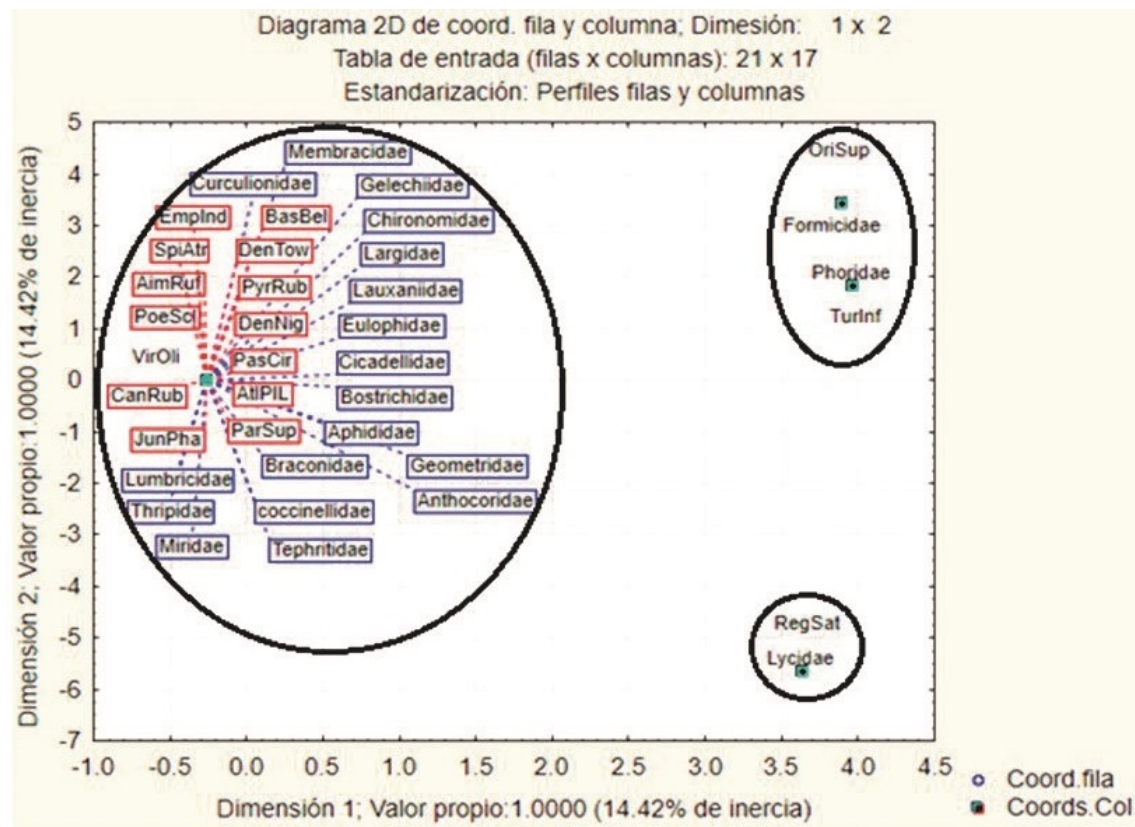

Figura 1. Representación gráfica de los resultados del ACS para las familias de insectos colectados por las aves durante su alimentación en las elevaciones 2600, 2800, y 3000.

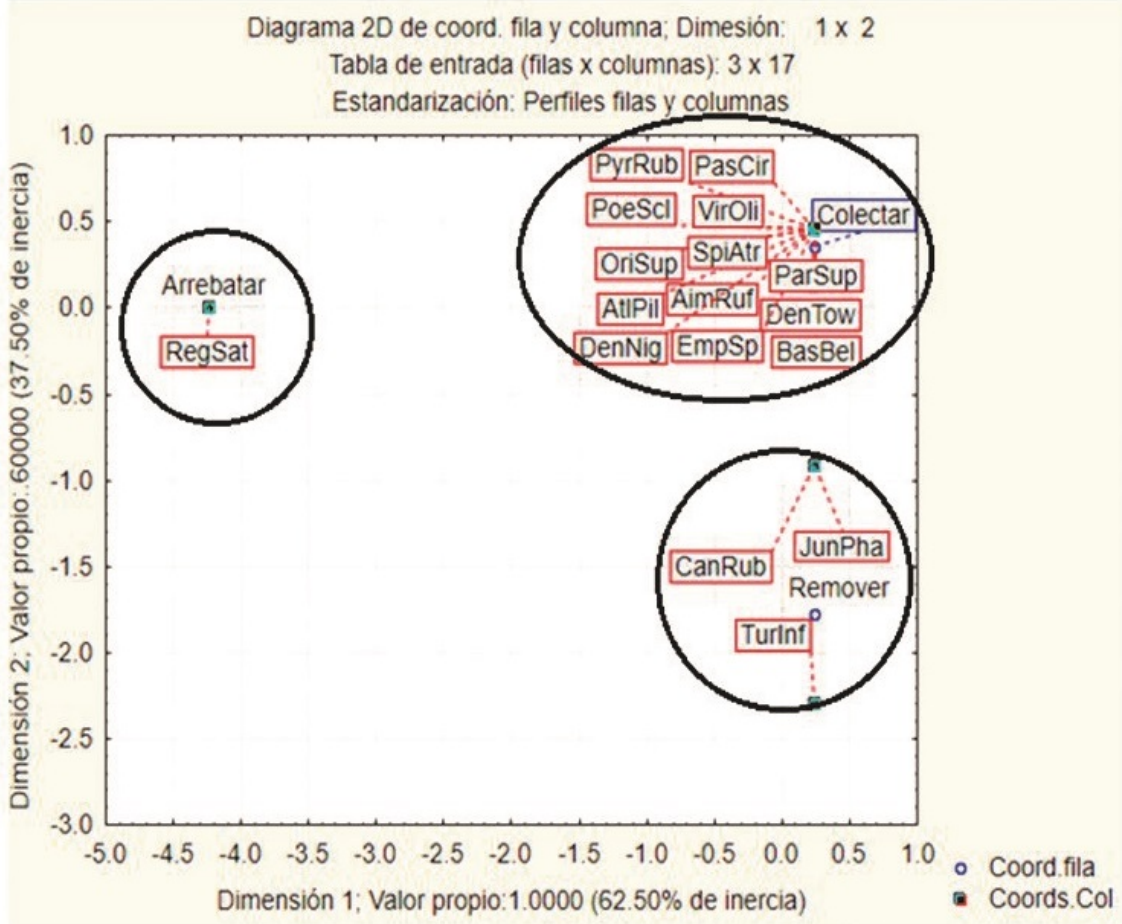

Figura 2. Representación gráfica de los resultados del ACS para las técnicas de cacería utilizadas por las aves durante su alimentación en las elevaciones 2600, 2800, y 3000. 
Romero-Díaz et al.

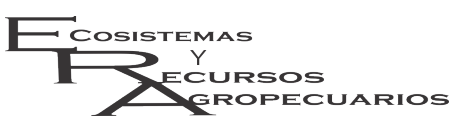

Coexistencia y segregación trófica de aves Ecosist. Recur. Agropec.

5(15):47r-489,2018

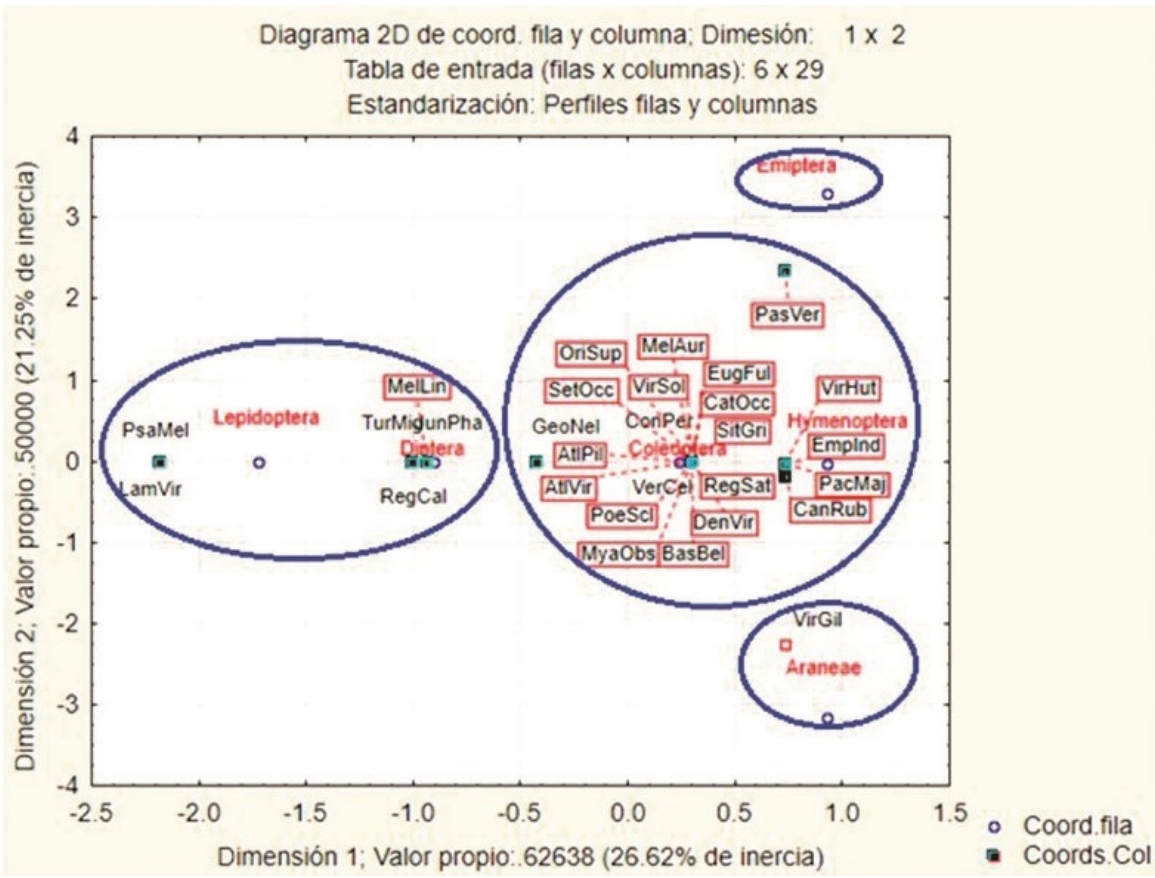

Figura 3. Representación gráfica de los resultados del ACS para los órdenes de insectos consumidos por las aves durante su alimentación en las elevaciones 2600, 2800 y 3000.

controlan algunos Coleópteros considerados plaga.Sobre lo mismo Figueroa y Corales (2003) reportan que las presas más ofrecidas a los críos de Picoides lignarius fueron los Coleópteros. En contraste Gámez-Virués et al. (2010) encontraron que la mayor disponibilidad de insectos presentes en estratos arbóreos son Araneae y Formicidae. Al respecto Gámez-Virués et al. (2007) señalan que el estudio del espectro trófico en la determinación de las dietas en aves insectívoras podría coadyuvar en el conocimiento de su historia natural con fines de manejo del hábitat.

Las tendencias evaluadas en los comportamientos alimenticios, coexistencia y segregación trófica exhibidos por las aves del presente estudio, coincide con lo reportado por Chávez-Villavicencio et al. (2012) quienes estudiaron la segregación de aves insectívoras con base a la morfometría del pico y su longitud total, hallando que la competencia interespecífica hace que las especies puedan coexistir en simpatría, considerando la distancia ecológica de segregación que disminuye la competencia, lo que hace que las aves coexistan. Sobre lo mismo Pacheco y Castilla (2000) demostraron que dos especies de aves ostreras coexisten debido a que una consume presas de textura blanda y la otra dura, por lo que no hay competencia. En contraste, Velasco (2016) señala que los estratos suelen ser una variable que podría o no incidir sobre la distribución de insectos y por lo tanto, sobre su distribución y consumo, impactando de diversas formas la coexistencia y segregación trófica en este grupo taxonómico. Al respecto Peña et al. (2010) mencionan que la especialización del hábitat y procesos como la dispersión, podrían ser un factor determinante en la coexistencia de especies. Al respecto, Chablé-Santos et al. (2012) evaluaron el efecto trófico de Streptopelia decaocto sobre especies nativas de Yucatán, encontrando segregación por competencia de alimento, espacios de anidación y por portador virus que afecta a las aves nativas. 
Romero-Díaz et al. Coexistencia y segregación trófica de aves Ecosist. Recur. Agropec. 5(15):4777-489,2018

\section{CONCLUSIONES}

Se determinó la composición de las dietas y coexistencia alimenticia en aves insectívoras de las elevaciones evaluadas. La comunidad avifaunística exhibe coexistencia y segregación trófica en la partición de los recursos alimenticios que les permite su ensamblaje en las elevaciones analizadas. Este es el primer estudio sobre la ecología básica alimenticia de este grupo en esta región estudiada. La altitud en bosques templados no es un factor determinante en la coexistencia.

\section{AGRADECIMIENTOS}

Al MC Prócoro Díaz Vargas por el apoyo económico para realizar la presente investigación, al C. Agustín Rodríguez Fuentes "Don Agus" por el apoyo en la identificación de insectos; a Daniel Alderete Rojas por la edición del mapa del área de estudio, a los Biólogos Fátima Berenice Sosa Villa nueva y Uriel Marcos Rivera por su apoyo en la realización de los trabajos en campo, a las autoridades de la comunidad de San Pablo Ixayoc por permitir realizar el proyecto.

\section{LITERATURA CITADA}

Adamik P, Korňan M (2004) Foraging ecology of two bark foraging passerine birds in an old-growth temperate forest. Ornis Fennica 81: 13-22.

Aguilar JM, Tinoco BA (2017) Ecología de polinización de Axinaea merianiae en los altos Andes del sur del Ecuador: Características de su néctar y aves polinizadoras. Avances en Ciencias e Ingenierías 1: 1-6.

Akaike H (1969) Fitting autoregresive models for prediction. Annals of the Institute of Statistical. Mathematics 21:243-247.

Berlanga H, Gómez de Silva H, Vargas-Canales VM, Rodríguez-Contreras V, Sánchez-González LA, OrtegaÁlvarez R, et al. (2015) Aves de México: Lista actualizada de especies y nombres comunes. CONABIO, México $122 p$

BirdLife International (2008) El estado de conservación de las aves del mundo: indicadores en tiempos de cambio. Cambridge, UK: BirdLife International. Wellbrook Court, Girton Road, Cambridge CB3 ONA, Reino Unido. 28p.

Borror DJ, Triplehorn A, Johnson NF (1989) An introduction to the study of insects. 6th ed. Harcourt Brace College Publishing. Orlando, Florida, USA. 875p.

Caicedo-Argüelles A, Cruz-Bernate L (2014) Actividades diarias y uso de hábitat de la reinita amarilla (Setophaga petechia) y la piranga roja (Piranga rubra) en un área verde urbana de Cali, Colombia. Ornitologia Neotropical 25: 247-260.

Camphuysen CJ, Webb A (1999) Multi-species feeding associations in North Sea seabirds: jointly exploiting a patchy environment. Ardea 87: 177-198.

Chablé-Santos J, Gómez-Uc E, Hernández-Betancourt S (2012) Registros reproductivos de la paloma de collar (Streptopelia decaocto) en Yucatán, México. Huitzil 13: 1-5.

Chávez-Villavicencio C, Séenz-Bolaños C, Spínola-Parallada M (2012) Segregación en aves insectívoras con base en la morfometría del pico y la longitud total. Aporte Santiaguino 5: 60-67.

Colorado ZG (2004) Relación de la morfometría de aves con gremios alimenticios. Boletín SAO 14: 25-32.

Cruz-Miranda Y, Ugalde-Lazama S, Tarango-Arámbula LA, Rosas-Rosas OC, Buendía-Espinoza JC, LozanoCavazos EA (2017) Modelo alternativo para determinar coexistencia y segregación trófica de dos felinos simpátricos: Puma concolor L. y Panthera onca L. Agroproductividad 5: 18-27. 
Cueto VR, López de Casanave J (2002) Foraging Behavior and Microhabitat Use of Bird Inhabiting Coastal Woodlands in Eastcentral Argentina. The Wilson Bulletin 114: 342- 348.

De la Ossa J, De la Ossa-Lacayo A, Monroy-Pineda M (2017) Abundance of domestic dove (Columbia livia domestica Gmelin, 1789) in Santiago de Tolú, Sucre, Colombia. Revista MVZ Córdoba 22: 5718-5727.

Equihua MA (1989) Estados inmaduros de los insectos. Instituto de Fitosanidad. Programa de Entomología. Colegio de Posdraduados, Campus Montecillo. 299p.

Fandiño B, Fernández JM, Thomann ML, Cajade R, Hernando AB (2016) Comunidades de aves de bosques y pastizales en los afloramientos rocosos aislados del Paraje Tres Cerros, Corrientes, Argentina. Revista de Biología Tropical 65: 535-550.

Figueroa RR, Corales SE (2003) Notas sobre la conducta de crianza del Carpintero Bataraz Grande (Picoides lignarius) en el bosque lluvioso templado del sur de Chile. Hornero 18: 119-122.

Frere E, Quintana F, Gandini P (2005) Cormoranes de la costa Patagónica: Estado poblacional, Ecología y Conservación. Hornero 20: 35-52.

Gallina S, López-González C (2011) Manual de técnicas para el estudio de la fauna. Volumen I. Universidad Autónoma de Querétaro-Instituto de Ecología. Querétaro, México. 377p.

Gámez-Virués S, Gurr GM, Raman A, Nicol HI (2010) Plant diversity and structure affect tree growth, herbivory and natural enemies in shelterbelts. Basic and Applied Ecology 11: 542-549.

Gámez-Virués S, Ronald SB, Geoff MG, Cilla K, Anantanarayanan R, Helen IN (2007) Arthropod prey of shelterbelt-associated birds: linking faecal samples with biological control of agricultural pests. Australian Journal of Entomology 46: 325-331.

Ginnobili S, Roffe A (2016) Dos usos de los modelos de optimalidad en las explicaciones por selección natural. Metatheoria 8: 43-55.

Haro JG (1998) Alimentacion de aves insectívoras en un bosque de coníferas en Río de los Sauces, (Cordoba, Argentina). Natura Neotropicalis 29: 117-125.

Johnson MD, Sherry TW, Strong AM, Medori A (2005) Migrants in Neotropical bird communities: an assessment of the breeding currency hypothesis. Journal of Animal Ecology 74: 333-341.

Mills KL (1998) Multispecies seabird feeding flocks in the Galapagos Islands. The Condor 100: 277-285

Moreno E (2007) Características territoriales, ambientales y sociopolíticas del Municipio de Texcoco, Estado de México Quivera 9: 177-206.

National Geographic Society (2002) Field guide to the birds of North América. Fourth edition. National Geographic Society, Washington, D.C. 480p.

Navarro-Sigüenza AG, Rebón-Gallardo MF, Gordillo-Martínez A, Townsend PA, Berlanga-García H, SánchezGonzález LA (2014) Biodiversidad de aves en México. Revista Mexicana de Biodiversidad 85: S476S495.

Ortiz-Pulido R, Alcántara-Carbajal JL, de la Cueva H, Martínez-Gómez J, Escalante P, de la Parra-Martínez SM, Feria TP, Albert S (2016) Conservación de aves en México, una instantánea de 2015. Huitzil 2: 234-238.

Ostrand WD (1999) Marbled murrelets as initiators of feeding flocks in Prince William Sound, Alaska. Waterbirds 22: 314-318. 
Pacheco C, Castilla J (2000) Ecología trófica de los ostreros Haematopus palliatus pitanay. Revista Chilena de Historia Natural 73: 533-541

Peña MA, Cardenaz D, Duque A (2010) Distribución de especies y su relación con la variación ambiental y espacial a escala local en un bosque de tierra firme en la Amazonia colombiana. Actualidades Biológicas 32: $41-51$.

Perovic DJ, Gámes-Virués S, Landis DA, Wackers GM, Gurr GM, Min-Sheng Y, Desneux N (2018) Managing biological control services through multi-trophic interactions: review and guidelines for implementation at local and lasdscape scales. Biological reviews 93: 306-321.

Peterson RT, Chalif EL (1989) Aves de México. Guía de campo. Editorial Diana. México. 473p.

Pineda-Pérez FE, Ugalde-Lezama S, Tarango-Arámbula LA, Lozano-Osornio A, Cruz-Miranda Y (2014) Ecología trófica de aves insectívoras en un área natural protegida de San Luis Potosí, México. Agroproductividad 7: 8-10.

Recher HF, Majer JD (2006) Effects of bird predation on canopy arthropods in Wandoo Eucalyptus wandoo Woodland. Austral Ecology 31: 349-360.

Rosas-Espinoza VC, Maya-Elizarraras E, Reyna-Bustos OF, Huerta-Martínez FM (2008) Diet of acorn woodpeckers at la primavera forest, Jalisco, Mexico. The Wilson Journal of Ornithology 120: 494-498.

Seeholzer G, Claramunt S, Brumfield R (2017) Niche evolution and diversification in a Neotropical radiation of birds (Aves: Furnariidae). International Journal of Organic Evolution 71: 702-715.

SEMARNAT (2010) NOM-059-SEMARNAT Protección ambiental-especies nativas de México de flora y fauna silvestres-Categorías de riesgo y especificaciones para su inclusión, exclusión o cambio-Listade especies en riesgo. Diario Oficial de la Federación. Secretaria de Medio Ambiente y Recursos Naturales. México. 78p.

Stehr FW (1991) Immature insects. Vol. 2. Kendall, Hunt Publishing Company. USA. 975p.

Torrens YA, Hernández FR, Barrero H (2017) Diversidad de aves residentes permanentes asociadas a un pinar natural de Pinus tropicalis y su relación con la estructura vertical de la vegetación. Revista Cubana de Ciencias Forestales 6: 31-44.

Ugalde-Lezama S, Tarango-Arámbula LA, Ramírez-Valverde G, Equihua-Martínez A, Valdez-Hernández JI (2011) Coexistencia trófica de aves carpinteras (Picidae) en un bosque de Pinus cembroides (Zucc.) del área natural protegida Peña Alta, San Diego de la Union, Guanajuato. Revista Chapingo Serie Ciencias Forestales y del Ambiente 17: 361-377.

Velasco AC, Talabante C, Viejo JL (2017) Estudio de selección del hábitat de la especie exótica bengalí rojo Amandava amandava, Linnaeus, 1758 en Iberia central (Aves: Paseriformes). Boletín de la Real Sociedad Española de Historia Natural Sección Biológica 3: 85-94.

Velasco PK (2016) Segregación estructural, climática y temporal de cinco especies simpátricas de Anolis (Sauria: Dactyloidae) en Boca de Canasí, Cuba/Structural, climatic and temporary segregation of five simpatric species of Anolis (Sauria: Dactyloidae) en Boca de Canasí, Cuba. Revista Cubana de Zoología 502: 49-54.

Villaseñor GJF, Santana E (2002) El monitoreo de poblaciones: herramienta necesaria para la conservación de aves en México. En: Gómez DH, Oliveras DA (eds.). Conservación de aves. Experiencias en México. CIPAMEX. México. pp: 224-262. 
Whitaker JO (1988) Food habits analysis of insectivorous bats. In: Kunz TH (ed.) Ecological and behavioral methods for the study of bats. Smithsonian Institution Press., Washington, D.C. pp 171-189. 
